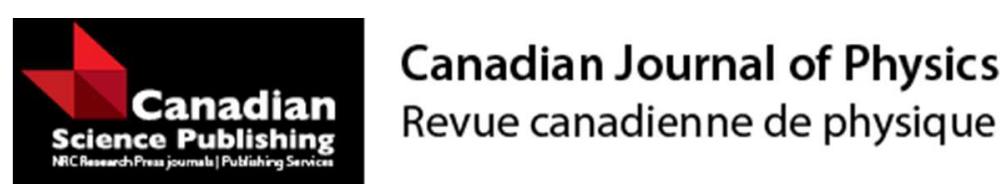

\title{
Bulk viscous string cosmological models in $f(R)$ gravity
}

\begin{tabular}{|r|l|}
\hline Journal: & Canadian Journal of Physics \\
\hline Manuscript ID & cjp-2017-0256.R1 \\
\hline Danuscript Type: & Article \\
\hline Complete List of Authors: & $\begin{array}{l}\text { Vijaya Santhi, M.; Andhra University, Applied Mathematics } \\
\text { Rao, V.U.M.; Andhra University, Applied Mathematics } \\
\text { Aditya, Y.; Andhra University, Applied Mathematics }\end{array}$ \\
\hline Keyword: & $\begin{array}{l}\text { Bianchi type-III metric, bulk viscosity, f(R) gravity, modified gravity, } \\
\text { cosmic strings }\end{array}$ \\
\hline $\begin{array}{r}\text { Is the invited manuscript for } \\
\text { consideration in a Special } \\
\text { Issue? : }\end{array}$ & N/A \\
\hline \multicolumn{2}{|l}{} \\
\hline
\end{tabular}

SCHOLARONE $^{\text {m }}$

Manuscripts 


\title{
Bulk viscous string cosmological models in $f(R)$ gravity
}

\author{
M.Vijaya Santhi, V.U.M.Rao, Y.Aditya \\ Department of Applied Mathematics, Andhra University, Visakhapatnam-530003, India \\ gv.santhi@live.com
}

\begin{abstract}
In this paper, we derive $f(R)$ gravity field equations with the help of a spatially homogeneous and anisotropic Bianchi type- $I I I$ space-time, in the presence of a bulk viscous fluid, containing one-dimensional cosmic strings. Here we obtained the solutions of the field equations to both, in the presence and in the absence of cosmic strings under some specific plausible physical conditions. In particular, cosmological models with bulk viscous strings in $f(R)$ theory of gravity, are obtained by using special law of variation for Hubble's parameter proposed by Berman (Nuovo Cimento B74:182, 1983). Various physical and kinematical properties of the models are, also, discussed.
\end{abstract}

Key words: Bianchi type- $I I I$ metric, bulk viscosity, $f(R)$ gravity, modified gravity, cosmic strings.

\section{Introduction}

It is clear from different observational data (Riess et al. [1]; Perlmutter et al. [2]) that the expansion of the universe is accelerating. The reason behind this observed acceleration is unknown, and is usually referred to as "dark energy problem". To address this problem, two different approaches have been advocated: one is to construct different dark energy candidates and the other is the modification of Einstein's theory of gravitation. Among various modified theories of gravitation, $f(R)$ theory of gravity is significant in which a general function of the Ricci scalar, $f(R)$, replaces $R$ in the standard Einstein-Hilbert Lagrangian. This provides an easy unification of early time inflation and late time acceleration [3]. The theory also gives a natural gravitational alternative to dark energy. Nojiri and Odintsov [4] and Capozziello and Laurentis [5] have studied many aspects on $f(R)$ gravity.

Friedmann-Robertson-Walker (FRW) models, being spatially homogeneous and isotropic in nature, are best for the representation of the large scale structure of the present universe. However, it is believed that the early universe may not have been exactly uniform. It is also 
found that some large-angle anomalies [6] appear in cosmic microwave background (CMB) radiations which violate the statistical isotropy of the universe. Bianchi type models, which are homogeneous but not necessarily isotropic seem to be the most promising explanation of these anomalies. Jaffe et al. [7] explored that removing a Bianchi component from the Wilkinson Microwave Anisotropy Probe (WMAP) data can account for several large-angle anomalies leaving the universe to be isotropic. Thus, the models with anisotropic background are the most suitable to describe the early stages of the universe. Bianchi type models are among the simplest models with anisotropic background. Recently, Bianchi type dark energy cosmological models in various theories of gravitation have been widely discussed in literature [8]-[14].

Shamir [15] studied exact vacuum solutions of Bianchi type I, III and Kantowski-Sachs space-times in the metric version of $f(R)$ gravity. Shamir [16] explored plane symmetric vacuum solutions of Bianchi type- $I I I$ cosmology in $f(R)$ gravity. Sharif and Shamir [17] discussed non-vacuum Bianchi types- $I$ and $V$ in $f(R)$ gravity. Sharif and Kausar [18] obtained anisotropic fluid and Bianchi type $I I I$ model in $f(R)$ gravity. Yilmaz et al. [19] studied quark and strange quark matter in $f(R)$ gravity for Bianchi type $I$ and $V$ spacetimes. Sharif and Arif [20] explored non-vacuum static cylindrically symmetric solution and energy distribution in $f(R)$ gravity. Sheykhi [21] studied magnetic strings in $f(R)$ gravity. Katore [22] discussed Bianchi type- $I I, V I I I$ and $I X$ string cosmological models in $f(R)$ gravity.

Sting cosmological model are important during structure formation in the early stages of evolution of the universe. Spontaneous symmetry breaking through the phase transition in the early universe result in a random network of stable line like topological defects familiar as cosmic strings. It is generally known that massive strings serve as starting point for the large structures like cluster of galaxies in the universe. So these string models have gained considerable attention of research workers. Stachel [23], Letelier [24], Vilenkin et al. [25], Banerjee et al. [26], Reddy [27], Rao et al. [28]-[29], Tripathy et al. [30] are some of the authors who have investigated several important aspects of string cosmological models in various theories of gravitation. Recently, Rao and Paparao [31] studied Bianchi type- $V$ string cosmological models in $f(R, T)$ gravity. Goswami et al. [32] explored anisotropic string cosmological models in Heckmann-Schucking space-time. Sahoo et al. [33] discussed Bianchi type string cosmological models in $f(R, T)$ gravity.

During the neutrinos coupling in the early stages of universe, the matter behaved as a 
viscous fluid. The coefficient of viscosity decreases as the universe expands. The effect of viscosity on the evolution of the universe and the strong dissipation, due to the neutrino viscosity, may considerably reduce the anisotropy of the black body radiation have been discussed by Misner [34]-[35]. Barrow [36], Padmanabhan and Chitre [37], Pavon et al. [38], Lima et al. [39] and Martens [40] are some of the authors have studied the possibility of bulk viscosity leading to inflationary-like solution in general relativistic FRW models. The effective total negative pressure which leads to a repulsive gravity in bulk viscosity overcomes the attractive gravity of the matter and gives an impulsion for rapid expansion of the universe. Many authors like Bali and Pradhan [41], Tripathy et al. [42], Singh and Kale [43], Rao and Sireesha [44] and Shri Ram et al. [45] have explored cosmological models with bulk viscosity in different theories of gravitation. Rao et al. [46]-[47] studied FRW bulk viscous cosmological model in some scalar-tensor theories of gravitation.

Tripathy et al. [30] studied string cloud cosmologies for Bianchi type- III models with electromagnetic field. Adhav et al. [48] discussed Bianchi type- $I I I$ string cosmological models in general theory of relativity. Rao et al. [49] obtained anisotropic universe with cosmic strings and bulk viscosity in a scalartensor theory of gravitation. Sagar et al. [50] have explored Bianchi type- $I I I$ bulk viscous cosmic string models in Brans-Dicke theory of gravitation.

Motivated by above investigations, in this paper, we focus our attention on the Bianchi type $I I I$ bulk viscous string model in metric $f(R)$ theory of gravity. The plan of the paper is follows: In sect. 2, we derive $f(R)$ field equations for Bianchi type- $I I I$ metric in the presence of bulk viscous fluid with one dimensional string. In sect. 3.2, bulk viscous string models are obtained by using Berman's law [51] for Hubble's parameter. Sect. 3.3 devoted to bulk viscous model (absence of string) in $f(R)$ gravity. Some physical parameters are also evaluated for our models. In the last section, we summarize the results.

\section{$2 \quad$ Metric and field equations}

We consider the spatially homogeneous and anisotropic Bianchi type- $I I I$ space-time as

$$
d s^{2}=d t^{2}-X^{2}(t) d x^{2}-Y^{2}(t) e^{2 b x} d y^{2}-Z^{2}(t) d z^{2}
$$

where $X, Y$ and $Z$ are functions of cosmic time $t$ only and $b$ is a positive constant. 
The field equations of $f(R)$ gravity are obtained from the action

$$
S=\int \sqrt{-g}\left(\frac{1}{16 \pi G} f(R)+L_{m}\right) d^{4} x,
$$

where $f(R)$ is a general function of the Ricci scalar and $L_{m}$ is the matter Lagrangian. Variation of action (2) with respect to metric gives the following field equations

$$
F(R) R_{i j}-\frac{1}{2} f(R) g_{i j}-\nabla_{i} \nabla_{j} F(R)+g_{i j} \square F(R)=\kappa T_{i j}
$$

where $F(R)=\frac{d f}{d R}$ and $\square=\nabla^{i} \nabla_{i}, \nabla_{i}$ is the covariant derivative. Contracting the field equations (3), we get

$$
F(R) R-2 f(R)+3 \square F(R)=\kappa T
$$

Using above equation in equation (3), the field equations take the form

$$
F(R) R_{i j}-\nabla_{i} \nabla_{j} F(R)-\kappa T_{i j}=g_{i j}\left(\frac{F(R) R-\square F(R)-\kappa T}{4}\right)
$$

Equation (4) is an important relationship between $f(R)$ and $F(R)$ which will be used to simplify the field equations and to evaluate $f(R)$.

The energy-momentum tensor for a bulk viscous fluid containing one-dimensional cosmic strings is given by

$$
\begin{aligned}
T_{i j} & =(\rho+\bar{p}) u_{i} u_{j}-\bar{p} g_{i j}-\lambda x_{i} x_{j} \\
\bar{p} & =p-3 \xi H(=\omega \rho)
\end{aligned}
$$

where $p=\omega_{0} \rho\left(0 \leq \omega_{0} \leq 1\right)$. Here $\bar{p}$ is the total pressure which includes the proper pressure $p, \lambda$ is the string tension density, $\rho$ is the rest energy density of the system, $\xi(t)$ is the coefficient of bulk viscosity, $3 \xi H$ is generally known as bulk viscous pressure, $H$ is the Hubble parameter of the model and $\omega=\omega_{0}-\zeta$ (where $\omega_{0}$ and $\zeta$ are constants). We consider $\rho, \bar{p}$ and $\lambda$ as functions of time $t$ only.

Also, $u_{i}$ is the four velocity vector, $x_{i}$ is a space-like vector which represents the anisotropic directions of the string and they satisfies

$$
g^{i j} u_{i} u_{j}=-x^{i} x_{j}=1, \quad u^{i} x_{i}=0
$$

We assume the string to be lying along the $z$-axis. The one dimensional strings are assumed to be loaded with particle and energy density is $\rho_{p}=\rho-\lambda$. 
Using comoving coordinates, field equations (5) for metric (1) yield the following equations:

$$
\begin{aligned}
\left(\frac{\ddot{X}}{X}+\frac{\dot{X} \dot{Y}}{X Y}+\frac{\dot{X} \dot{Z}}{X Z}-\frac{b}{X^{2}}\right) F-\frac{1}{2} f(R)+\ddot{F}+\dot{F}\left(\frac{\dot{Y}}{Y}+\frac{\dot{Z}}{Z}\right) & =-\kappa \bar{p} \\
\left(\frac{\ddot{Y}}{Y}+\frac{\dot{X} \dot{Y}}{X Y}+\frac{\dot{Y} \dot{Z}}{Y Z}-\frac{b}{X^{2}}\right) F-\frac{1}{2} f(R)+\ddot{F}+\dot{F}\left(\frac{\dot{X}}{X}+\frac{\dot{Z}}{Z}\right) & =-\kappa \bar{p} \\
\left(\frac{\ddot{Z}}{Z}+\frac{\dot{X} \dot{Z}}{X Z}+\frac{\dot{Y} \dot{Z}}{Y Z}\right) F-\frac{1}{2} f(R)+\ddot{F}+\dot{F}\left(\frac{\dot{X}}{X}+\frac{\dot{Y}}{Y}\right) & =\kappa(\lambda-\bar{p}) \\
\left(\frac{\ddot{X}}{X}+\frac{\ddot{Y}}{Y}+\frac{\ddot{Z}}{Z}\right) F-\frac{1}{2} f(R)+\dot{F}\left(\frac{\dot{X}}{X}+\frac{\dot{Y}}{Y}+\frac{\dot{Z}}{Z}\right) & =\kappa \rho \\
F\left(\frac{\dot{X}}{X}-\frac{\dot{Y}}{Y}\right) & =0
\end{aligned}
$$

where overhead dot stands for ordinary differentiation with respect to $t$.

\section{Solutions of the field equations}

Integrating equation (13) we obtain

$$
X=k Y
$$

where $k$ is an integration constant, which can be chosen as unity without loss of any generality, so that we get

$$
X=Y
$$

Now, using equation (15), the field equations (9)-(13) reduce to the following independent equations:

$$
\begin{aligned}
\left(\frac{\ddot{X}}{X}+\frac{\dot{X}^{2}}{X^{2}}+\frac{\dot{X} \dot{Z}}{X Z}-\frac{b}{X^{2}}\right) F-\frac{1}{2} f(R)+\ddot{F}+\dot{F}\left(\frac{\dot{X}}{X}+\frac{\dot{Z}}{Z}\right) & =-\kappa \bar{p} \\
\left(\frac{\ddot{Z}}{Z}+2 \frac{\dot{X} \dot{Z}}{X Z}\right) F-\frac{1}{2} f(R)+\ddot{F}+2 \dot{F} \frac{\dot{X}}{X} & =\kappa(\lambda-\bar{p}) \\
\left(2 \frac{\ddot{X}}{X}+\frac{\ddot{Z}}{Z}\right) F-\frac{1}{2} f(R)+\dot{F}\left(\frac{2 \dot{X}}{X}+\frac{\dot{Z}}{Z}\right) & =\kappa \rho .
\end{aligned}
$$


We define the following parameters for the Bianchi type- III model:

Spatial volume and average scale factor of the model

$$
V=\sqrt{-g}=X^{2} Z, \quad a(t)=\left(X^{2} Z\right)^{\frac{1}{3}}
$$

Anisotropic parameter $A_{h}$ is given by

$$
A_{h}=\frac{1}{3} \sum_{i=1}^{3}\left(\frac{H_{i}-H}{H}\right)^{2} .
$$

where $H_{1}=H_{2}=\frac{\dot{X}}{X}, H_{3}=\frac{\dot{Z}}{Z}$ are directional Hubble's parameters and $H=\frac{1}{3}\left(\frac{2 \dot{X}}{X}+\frac{\dot{Z}}{Z}\right)$ is mean Hubble's parameter.

Expansion scalar and shear scalar are defined as

$$
\begin{gathered}
\theta=u_{; i}^{i}=\frac{2 \dot{X}}{X}+\frac{\dot{Z}}{Z} \\
\sigma^{2}=\frac{1}{2} \sigma^{i j} \sigma_{i j}=\frac{1}{3}\left(\frac{\dot{X}}{X}-\frac{\dot{Z}}{Z}\right)^{2} .
\end{gathered}
$$

Deceleration parameter is given by

$$
q=\frac{d}{d t}\left(\frac{1}{H}\right)-1
$$

\subsection{Bulk viscous string model $(\lambda \neq 0)$}

In this case set of equations (16)-(18) forms a system of three independent equations with six unknowns $X, Z, F(R), \bar{p}, \rho$ and $\lambda$. Hence to find a determinate solution of these highly non-linear differential equations we use the following physically viable conditions:

(i) The shear scalar $\sigma$ is proportional to scalar expansion $\theta$, which leads to a relationship between the metric potentials, so that we can take [52]

$$
X=Z^{n}
$$

where $n \neq 0$ is a constant and preserves the anisotropic character of the space-time.

(ii) $f(R)$ theory of gravity has been shown equivalent to scalar-tensor theory of gravity that is incompatible with solar system tests of general relativity, as long as the scalar 
field propagates over solar system scales [53]. The power-law relation between scalar field and average scale factor has already been used by Johri and Sudharsan [54] in the context of FRW models with bulk viscosity in BransDicke theory. However, Uddin et al. [55] have established a result in the context of $f(R)$ theory of gravity which shows that

$$
F(R) \propto[a(t)]^{m}(25) \text { where } m \text { is an arbitrary }
$$

constant. Thus using power-law relation between $F$ and $a$, we have

$$
F(R)=F_{0}[a(t)]^{m}
$$

where $F_{0}$ is proportionality constant.

(iii) The Hubble parameter $H$ proposed by Berman [51] defined as

$$
H=\beta[a(t)]^{-\alpha}
$$

where $\beta>0, \alpha \geq 0$ are constants and $a(t)$ is average scale factor. This relation gives a constant value of deceleration parameter.

By solving equation (27), we obtain

$$
\begin{array}{ll}
a(t)=\left(\alpha \beta t+c_{3}\right)^{\alpha}, & \alpha \neq 0, \\
a(t)=c_{2} \exp (\beta t), & \alpha=0 .
\end{array}
$$

Thus the above two values of average scale factor corresponding to two different models of the universe.

\subsubsection{Model with power law expansion $(\alpha \neq 0)$}

From equations (15), (24) and (28), we get the metric coefficients for power law expansion model of the universe as

$$
X=Y=\left(\alpha \beta t+c_{3}\right)^{\frac{3 n}{\alpha(2 n+1)}}, Z=\left(\alpha \beta t+c_{3}\right)^{\frac{3}{\alpha(2 n+1)}}
$$

From equations (24) and (28), the function $F(R)$ becomes

$$
F=F_{0}\left(\alpha \beta t+c_{3}\right)^{\stackrel{m}{\alpha}} .
$$


From equations (16)-(18), we get the string energy density and tension density

$$
\begin{gathered}
\rho=\frac{F_{0}\left(\alpha \beta t+c_{3}\right)^{\frac{m}{\alpha}}}{\kappa(1+\omega)}\left\{\frac{\beta^{2}\left[3\left(3(1-n)-\alpha\left(2 n^{2}+2 n+1\right)\right)\right]}{\left(\alpha \beta t+c_{3}\right)^{2}(2 n+1)^{2}}+\frac{m(3 n+(\alpha-m)(2 n+1))}{(2 n+1)\left(\alpha \beta t+c_{3}\right)^{2}}\right. \\
\left.+b\left(\alpha \beta t+c_{3}\right)^{\frac{-6 n}{\alpha(2 n+1)}}\right\} . \quad(32) \\
\lambda=\frac{F_{0}\left(\alpha \beta t+c_{3}\right)^{\frac{m}{\alpha}}}{\kappa}\left\{\frac{\beta^{2}\left[3\left(\left(2 n^{2}-n-1\right)(\alpha-3)+m(n-1)(2 n+1)\right)\right]}{\left(\alpha \beta t+c_{3}\right)^{2}(2 n+1)^{2}}+b\left(\alpha \beta t+c_{3}\right)^{\frac{-6 n}{\alpha(2 n+1)}}\right\} .
\end{gathered}
$$

The coefficient of bulk viscosity is given by

$$
\xi=\frac{\left(\omega_{0}-\omega\right) F_{0}\left(\alpha \beta t+c_{3}\right)^{\frac{m}{\alpha}-1}}{\kappa(1+\omega) \beta}\left\{\begin{array}{c}
\frac{\beta^{2}\left[3\left(3(1-n)-\alpha\left(2 n^{2}+2 n+1\right)\right)\right]}{\left(\alpha \beta t+c_{3}\right)^{2}(2 n+1)^{2}}+b\left(\alpha \beta t+c_{3}\right)^{\frac{-6 n}{\alpha(2 n+1)}} \\
+\frac{m(3 n+(\alpha-m)(2 n+1))}{(2 n+1)\left(\alpha \beta t+c_{3}\right)^{2}}
\end{array}\right\}
$$

We obtain the total pressure as

$$
\begin{aligned}
& \bar{p}=\frac{\omega F_{0}\left(\alpha \beta t+c_{3}\right)^{\frac{m}{\alpha}}}{\kappa(1+\omega)}\left\{\frac{\beta^{2}\left[3\left(3(1-n)-\alpha\left(2 n^{2}+2 n+1\right)\right)\right]}{\left(\alpha \beta t+c_{3}\right)^{2}(2 n+1)^{2}}+\frac{m(3 n+(\alpha-m)(2 n+1))}{(2 n+1)\left(\alpha \beta t+c_{3}\right)^{2}}\right. \\
& \left.+b\left(\alpha \beta t+c_{3}\right)^{\frac{-6 n}{\alpha(2 n+1)}}\right\} \text {. }
\end{aligned}
$$

The scalar curvature and $f(R)$ function are found to be

$$
\begin{aligned}
R & =2\left[\frac{\ddot{X}}{X}+\frac{\ddot{Y}}{Y}+\frac{\ddot{Z}}{Z}+\frac{\dot{X} \dot{Y}}{X Y}+\frac{\dot{X} \dot{Z}}{X Z}+\frac{\dot{Z} \dot{Y}}{Z Y}-\frac{b}{X^{2}}\right] \\
& =\frac{6 \beta^{2}\left[3\left(3 n^{2}+2 n+1\right)-\alpha\left(4 n^{2}+5 n+1\right)\right]}{(2 n+1)^{2}\left(\alpha \beta t+c_{3}\right)^{2}}-b\left(\alpha \beta t+c_{3}\right)^{\frac{-6 n}{\alpha(2 n+1)}}, \\
f(R)= & \frac{3 F_{0} \beta^{2}}{2\left(\alpha \beta t+c_{3}\right)^{2-\frac{m}{\alpha}}}\left\{\frac{\kappa\left(-2 n^{2}+n+1\right)(\alpha+\beta+m(n-1))}{(2 n+1)^{2}}+m(m-\alpha+3)\right. \\
& \left.-\kappa\left(\frac{1-3 \omega}{1+\omega}\right)\left(\frac{\left[\left(3(1-n)-\alpha\left(2 n^{2}+2 n+1\right)\right)\right]}{2(2 n+1)^{2}}+\frac{m(3 n+(\alpha-m)(2 n+1))}{6(2 n+1)}\right)\right\} \\
& -F_{0} b\left(\frac{1-\omega}{1+\omega}\right)\left(\alpha \beta t+c_{3}\right)^{\frac{m}{\alpha}-\frac{6 n}{\alpha(2 n+1)}}+\frac{R F_{0}}{2}\left(\alpha \beta t+c_{3}\right)^{\frac{m}{\alpha}} .
\end{aligned}
$$

Now metric (1) can be written as

$$
d s^{2}=d t^{2}-\left(\alpha \beta t+c_{3}\right)^{\frac{6 n}{\alpha(2 n+1)}}\left(d x^{2}+e^{2 x} d y^{2}\right)-\left(\alpha \beta t+c_{3}\right)^{\frac{6}{\alpha(2 n+1)}} d z^{2} .
$$

Thus, metric (38) together with (32)-(37) constitutes a Bianchi type-III anisotropic string cosmological model with bulk viscosity in $f(R)$ theory of gravity with the following parameters. 


\section{Properties of the model:}

The following properties are important in discussion of cosmology of obtained anisotropic bulk viscous string model:

Spatial volume of the model

$$
V=\left(\alpha \beta t+c_{3}\right)^{\frac{3}{\alpha}} e^{b x}
$$

Directional Hubble's parameters are

$$
H_{1}=H_{2}=\frac{3 n \beta}{(2 n+1)\left(\alpha \beta t+c_{3}\right)}, \quad H_{3}=\frac{3 \beta}{(2 n+1)\left(\alpha \beta t+c_{3}\right)} .
$$

Hubble's parameter is

$$
H=\frac{\beta}{\alpha \beta t+c_{3}}
$$

Expansion scalar and shear scalar are given by

$$
\theta=\frac{3 \beta}{\alpha \beta t+c_{3}}, \quad \sigma^{2}=\frac{3(n-1)^{2} \beta^{2}}{(2 n+1)^{2}\left(\alpha \beta t+c_{3}\right)^{2}} .
$$

Anisotropic parameter $A_{h}$ and deceleration parameter are given by

$$
\begin{gathered}
A_{h}=\frac{2(n-1)^{2}}{(2 n+1)^{2}} . \\
q=\alpha-1 .
\end{gathered}
$$

\subsubsection{Model with exponential expansion $(\alpha=0)$}

In this case, we get the average scale factor as

$$
a(t)=c_{2} \exp (\beta t)
$$

and from equations (15), (24) and (26), the scale factors and the function $F(R)$ as

$$
\begin{aligned}
X=Y & =\left[c_{2} \exp (\beta t)\right]^{\frac{3 n}{2 n+1}} \\
Z & =\left[c_{2} \exp (\beta t)\right]^{\frac{3}{2 n+1}} \\
F(R) & =F_{0}\left[c_{2} \exp (\beta m t)\right] .
\end{aligned}
$$

From equations (16)-(18), we get the string energy density and tension density

$$
\rho=\frac{F_{0} c_{2}^{m} \exp (\beta m t)}{\kappa(1+\omega)}\left\{\frac{9 \beta^{2}(1-n)}{(2 n+1)^{2}}-m \beta^{2}\left(\frac{m(2 n+1)-3 n}{2 n+1}\right)+b c_{2}^{\frac{-6 n}{2 n+1}} \exp \left(\frac{-6 n \beta t}{2 n+1}\right)\right\} .
$$




$$
\lambda=\frac{F_{0} c_{2}^{m} \exp (\beta m t)}{\kappa}\left\{\frac{9 \beta^{2}\left(1-2 n^{2}+n\right)}{(2 n+1)^{2}}+\frac{3 m \beta^{2}(n-1)}{2 n+1}+b c_{2}^{\frac{-6 n}{2 n+1}} \exp \left(\frac{-6 n \beta t}{2 n+1}\right)\right\} .
$$

The coefficient of bulk viscosity is given by

$$
\begin{aligned}
\xi=\frac{\left(\omega-\omega_{0}\right) F_{0} c_{2}^{m} \exp (\beta m t)}{3 \beta \kappa(1+\omega)}\left\{\frac{9 \beta^{2}(1-n)}{(2 n+1)^{2}}-\right. & m \beta^{2}\left(\frac{m(2 n+1)-3 n}{2 n+1}\right) \\
& \left.+b c_{2}^{\frac{-6 n}{2 n+1}} \exp \left(\frac{-6 n \beta t}{2 n+1}\right)\right\} .
\end{aligned}
$$

We obtain the total pressure as

$$
\begin{array}{r}
\bar{p}=\frac{\omega F_{0} c_{2}^{m} \exp (\beta m t)}{\kappa(1+\omega)}\left\{\frac{9 \beta^{2}(1-n)}{(2 n+1)^{2}}-m \beta^{2}\left(\frac{m(2 n+1)-3 n}{2 n+1}\right)\right. \\
\left.+b c_{2}^{\frac{-6 n}{2 n+1}} \exp \left(\frac{-6 n \beta t}{2 n+1}\right)\right\} .
\end{array}
$$

The scalar curvature $R$ and $f(R)$ function are found to be

$$
\begin{aligned}
R & =\frac{18 \beta^{2}\left[\left(3 n^{2}+2 n+1\right)\right.}{(2 n+1)^{2}}-b c_{2}^{\frac{-6 n}{\alpha(2 n+1)}} \exp \left(\frac{-6 \beta n t}{2 n+1}\right), \\
f(R)= & \frac{F_{0} c_{2}^{m} \exp (m \beta t)}{2}\left\{\frac{9 \beta^{2}}{(2 n+1)^{2}}\left(\left(1-2 n^{2}+n\right)+\frac{(1-3 \omega)(1-n)}{\omega+1}\right)\right. \\
& +\frac{2 b(1-\omega)}{1+\omega} \frac{c_{2}^{\frac{-6 n}{\alpha(2 n+1)}}}{\exp \left(\frac{6 \beta n t}{2 n+1}\right)}+\left(R+3 \beta^{2} m(m+3)\right) \\
& \left.+\frac{m \beta^{2}}{2 n+1}\left[\frac{3(n-1)(1+\omega)-(1-3 \omega)[m(2 n+1)-3 n]}{(2 n+1)(1+\omega)}\right]\right\} .
\end{aligned}
$$

Now metric (1) can be written as

$$
d s^{2}=d t^{2}-\left(c_{2}\right)^{\frac{6 n}{(2 n+1)}} \exp \left(\frac{6 n \beta t}{2 n+1}\right)\left(d x^{2}+e^{2 x} d y^{2}\right)-\left(c_{2}\right)^{\frac{6}{(2 n+1)}} \exp \left(\frac{6 \beta t}{2 n+1}\right) d z^{2}
$$

Thus, metric (38) together with (47)-(52) constitutes a Bianchi type- $I I I$ anisotropic string cosmological model with bulk viscosity in $f(R)$ theory of gravity with the following parameters.

\section{Properties of the model:}

Spatial volume of the model

$$
V=c_{2}^{3} \exp (3 \beta t+b x) .
$$


Directional Hubble's parameters, Hubble's parameter, expansion scalar are given by

$$
\begin{aligned}
H_{1}=H_{2} & =\frac{3 n \beta}{2 n+1}, \quad H_{3}=\frac{3 \beta}{2 n+1}, \\
H & =\beta \\
\theta & =3 \beta .
\end{aligned}
$$

Shear scalar, mean anisotropic parameter and deceleration parameter are respectively given by

$$
\begin{aligned}
\sigma^{2} & =\frac{3(n-1)^{2} \beta^{2}}{(2 n+1)^{2}} \\
A_{h} & =\frac{2(n-1)^{2}}{(2 n+1)^{2}} \\
q & =-1
\end{aligned}
$$

\subsection{Bulk viscous model $(\lambda=0)$}

In this case the field equations (16)-(18) reduce to

$$
\begin{aligned}
&\left(\frac{\ddot{X}}{X}+\frac{\dot{X}^{2}}{X^{2}}-\frac{\dot{X} \dot{Z}}{X Z}-\frac{\ddot{Z}}{Z}-\frac{b}{X^{2}}\right) F+\left(\frac{\dot{Z}}{Z}-\frac{\dot{X}}{X}\right) \dot{F}=0 \\
&\left(\frac{\ddot{X}}{X}-\frac{\dot{X}^{2}}{X^{2}}-\frac{\dot{X} \dot{Z}}{X Z}+\frac{\ddot{Z}}{Z}+\frac{b}{X^{2}}\right) F+\dot{F} \frac{\dot{X}}{X}=\kappa(\bar{p}+\rho) .
\end{aligned}
$$

Now equations (61) and (62) are two independent equations in five unknowns $A, B, \bar{p}, f(R)$ and $\rho$. Hence to find a determinate solution we use the physically possible conditions (i) and (ii) given in section 3.1 .

From equations (61), (24) and (26), we obtain the metric potentials as

$$
X=Y=\left[n\left(c_{1} t+c_{2}\right)\right]^{\frac{1}{n}}, \quad Z=n\left(c_{1} t+c_{2}\right)
$$

where $c_{1}=\sqrt{\frac{3 b}{(n-1)(n(3-2 m)+3-m)}}$ and $c_{2}$ is an integration constant.

From equations (26) and (63), the function $F(R)$ becomes

$$
F(R)=F_{0}\left[n\left(c_{1} t+c_{2}\right)\right]^{\frac{m(2 n+1)}{3 n}} .
$$

The metric (1) can now be written as

$$
d s^{2}=d t^{2}-\left[n\left(c_{1} t+c_{2}\right)\right]^{\frac{2}{n}}\left(d x^{2}+e^{2 b x} d y^{2}\right)-\left[n\left(c_{1} t+c_{2}\right)\right]^{2} d z^{2} .
$$


From equations (62)-(64), we get the energy density in the model as

$$
\rho=\frac{F_{0}\left[n\left(c_{1} t+c_{2}\right)\right]^{\frac{m(2 n+1)}{3 n}}}{3 \kappa \omega n^{2}\left(c_{1} t+c_{2}\right)^{2}}\left[b-2 c_{1}^{2} n(n+1)+\frac{m c_{1}^{2}(2 n+1)(n(1-2 m)-m-1)}{6}\right] .
$$

The coefficient of bulk viscosity is given by

$\xi=\frac{\left(\omega_{0}-\omega\right) F_{0}\left[n\left(c_{1} t+c_{2}\right)\right]^{\frac{m(2 n+1)}{3 n}}}{3(2 n+1) c_{1} \kappa \omega n\left(c_{1} t+c_{2}\right)}\left[b-2 c_{1}^{2} n(n+1)+\frac{m c_{1}^{2}(2 n+1)(n(1-2 m)-m-1)}{6}\right]$.

We obtain the total pressure as

$$
\bar{p}=\frac{F_{0}\left[n\left(c_{1} t+c_{2}\right)\right]^{\frac{m(2 n+1)}{3 n}}}{3 \kappa n^{2}\left(c_{1} t+c_{2}\right)^{2}}\left[b-2 c_{1}^{2} n(n+1)+\frac{m c_{1}^{2}(2 n+1)(n(1-2 m)-m-1)}{6}\right] .
$$

The scalar curvature $R$ and $f(R)$ function are found to be

$$
\begin{gathered}
R=\frac{2 c_{1}^{2}\left(2 n^{2}+n+1\right)-2 b}{n^{2}\left(c_{1}+c_{2}\right)^{2}}, \\
f(R)=\frac{F_{0}\left[n\left(c_{1} t+c_{2}\right)\right]^{\frac{m(2 n+1)}{3 n}}}{2 n^{2}\left(c_{1} t+c_{2}\right)^{2}}\left\{2 c_{1}^{2}\left(n^{2}+1+\frac{n(n+1)}{3 \omega}\right)-\frac{(3 \omega+b)}{3 \omega}\right. \\
\left.+\frac{m c_{1}^{2}(3 \omega+1)(2 n+1)(n(2 m-1)+m+1)}{18 \omega}\right\} .
\end{gathered}
$$

If $\omega=\omega_{0}$, then the coefficient of bulk viscosity $\xi=0$ (given in equation (67)). Thus, the model (65) together with (66)-(70) constitutes Bianchi type- $I I I$ perfect fluid model in $f(R)$ gravity for $\omega=\omega_{0}$. Surprisingly, this perfect fluid model is quite similar to the model obtained by Sharif and Kausar [18].

However, metric (65) along with (66)-(70) represents Bianchi type-III bulk viscous cosmological model in $f(R)$ gravity with the following parameters which are important in the discussion of the cosmological models.

Spatial volume and average scale factor of the model are

$$
\begin{aligned}
V & =\left[n\left(c_{1} t+c_{2}\right)\right]^{\frac{2 n+1}{n}} \\
a & =\left[n\left(c_{1} t+c_{2}\right)\right]^{\frac{2 n+1}{3 n}} .
\end{aligned}
$$

Directional Hubble's parameters are

$$
H_{1}=H_{2}=\frac{c_{1}}{c_{1} t+c_{2}}, \quad H_{3}=\frac{c_{1}}{n\left(c_{1} t+c_{2}\right)}
$$


Mean Hubble's parameter is

$$
H=\frac{(2 n+1) c_{1}}{3 n\left(c_{1} t+c_{2}\right)} .
$$

Expansion scalar and shear scalar are given by

$$
\begin{aligned}
\theta & =\frac{(2 n+1) c_{1}}{n\left(c_{1} t+c_{2}\right)}, \\
\sigma^{2} & =\frac{(n-1)^{2} c_{1}^{2}}{3 n^{2}\left(c_{1} t+c_{2}\right)^{2}} .
\end{aligned}
$$

Anisotropic parameter $A_{h}$ and deceleration parameter are given by

$$
\begin{aligned}
A_{h} & =\frac{2(n-1)^{2}}{(2 n+1)^{2}}, \\
q & =\frac{n-1}{2 n+1} .
\end{aligned}
$$

\section{Summary and Conclusions}

Here we have presented Bianchi type-III bulk viscous string cosmological models in metric $f(R)$ theory of gravity. We have obtained cosmological models corresponding to bulk viscous fluid and bulk viscous cosmic strings using some physically viable conditions.

Power-law model (38) of the universe has a point type singularity at $t^{*}=\frac{c_{3}}{\alpha \beta}$. Physical parameters like directional Hubble's parameters $\left(H_{1}, H_{2}\right.$ and $\left.H_{3}\right)$, mean Hubble's parameter $H$, expansion $(\theta)$ and shear scalars $\left(\sigma^{2}\right)$ are all infinite at this point for $\alpha>0$ but volume $(V)$ of the model vanishes. Because the mean anisotropy parameter $A_{h} \neq 0$, the model is anisotropic. String energy density $(\rho)$ and tension density $(\lambda)$, coefficient of bulk viscosity $(\xi)$, total pressure $(\bar{p})$ and Ricci scalar $(R)$ are also infinite while the metric potentials vanish at this singularity point for $m<2 \alpha$ and $(2 n+1) m<6 n$. As $t \rightarrow \infty$, the expansion and shear scalar decrease for $\alpha>0$ i.e., the rate of expansion slows down with time. The positive sign of $q$ corresponds to standard decelerating model whereas the negative sign indicates acceleration. For $\alpha>1$, deceleration parameter $q>0$, therefore the model represents a decelerating model whereas for $0<\alpha<1$, we get $-1 \leq q<0$, which describes an accelerating model of the universe. So we can conclude from these observations that the 
model starts its expansion from zero volume with infinite string densities and pressure at $t^{*}$ and it continues to expand with time. The exponential model $(53)(\alpha=0)$ is nonsingular model of the universe. The parameters $H_{1}, H_{2}, H_{3}, H, \theta$ and $\sigma^{2}$ are constants without depending on time. The metric potentials do not vanish for this model. In this case, we have accelerating model $(q=-1)$ of the universe. String densities and pressure become constants when $t=0$. However they are decreasing as $t \rightarrow \infty$. For $n=1$ and $b=0$, it is interesting to note that in the both (power law and exponential law) models, we have $\lambda=0, A_{h}=0$ and $\sigma^{2}=0$, i.e., in this case the strings in the universe do not survive and the models become isotropic and shear free.

We observe that the spatial volume of the bulk viscous model (65) increases with the growth of cosmic time which shows that we have an expanding model. At $t=\frac{-c_{2}}{c_{1}}$, the Hubble's parameters, the scalar expansion, the shear scalar, $f(R)$ function, the isotropic pressure, energy density and the bulk viscosity assume infinitely large values for $m<\frac{6 n}{2 n+1}$, whereas they all vanish as $t \rightarrow \infty$. The model accelerates when $q<0$, and if $q>0$, it decelerates in the standard way. For $n<1$, the deceleration parameter $q<0$, thus our model accelerates which is in accordance with the present day scenario of accelerating universe. Also, since the bulk viscosity $\xi$ decreases with time we get, ultimately, inflationary model. However, the average anisotropic parameter $A_{h}$ remains constant throughout the evolution of the universe and it becomes zero when $n=1$. Also the model free from initial singularity which supports the Murphy [56] result stated that the introduction of bulk viscosity avoids an initial singularity.

Acknowledgments: The authors are thankful to the reviewer for the constructive comments which have certainly improve the quality of the manuscript.

\section{References}

[1] A. Riess, et al.: Astron. J. 116, 1009 (1998).

[2] S. Perlmutter, et al.: Astrophys. J. 517, 565 (1999).

[3] S. Nojiri, S.D. Odintsov: Int. J. Geom. Meth. Mod. Phys. 4, 115 (2007).

[4] S. Nojiri, S.D. Odintsov: (2011) (preprint: 1011.0544). 
[5] S. Capozziello, M. Laurentis: Phys. Rep. 509, 1 (2011).

[6] H.K. Eriksen, et al.: Astrophys. J. 605, 1420 (2004).

[7] T.R. Jaffe, et al.: Astrophys. J. 643, 616 (2006).

[8] Y. Aditya, et al.: Astrophys Space Sci. 361, 56 (2016).

[9] M.V. Santhi, et al.: Astrophys Space Sci. 361, 142 (2016).

[10] M.V. Santhi, et al.: Can. J. Phys. 94, 578 (2016).

[11] D.R.K. Reddy, et al.: Astrophys Space Sci 361, 349 (2016).

[12] M.V. Santhi, et al.: Int J Theor Phys 56, 362 (2017).

[13] M.V. Santhi, et al.: Can. J. Phys. 95, 381 (2017).

[14] M.V. Santhi, et al.: Can. J. Phys. 95, 179 (2017).

[15] M.F. Shamir: Astrophys Space Sci. 330, 183 (2010).

[16] M.F. Shamir: Int J Theor Phys 50, 637 (2011).

[17] M. Sharif, M.F. Shamir: Gen Relativ Gravit 42, 2643 (2010).

[18] M. Sharif, H.R.Kausar: Physics Letters B 697, 1 (2011a).

[19] I. Yilmaz, et al.: Gen Relativ Gravit 44, 2313 (2012).

[20] M. Sharif, S. Arif: Astrophys Space Sci. 342, 237 (2012).

[21] A. Sheykhi: Gen. Relativ Gravit, DOI 10.1007/s10714-012-1388-6 (2012).

[22] S.D. Katore: Int J Theor Phys 54, 2700 (2015).

[23] J. Stachel: Phys. Rev. D 21, 2171 (1980).

[24] P.S. Letelier: Phys. Rev. D 28, 2414 (1983).

[25] A. Vilenkin, et al.: Three Hundred Years of Gravitation p. 499. Cambridge Univ. Press, Cambridge (1987). 
[26] A. Banerjee, et al.: Pramana 34, 1 (1990).

[27] D.R.K. Reddy: Astrophys. Space Sci. 286, 356 (2003).

[28] V.U.M. Rao, et al.: Astrophys Space Sci 314, 73 (2008).

[29] V.U.M. Rao, et al.: Astrophys Space Sci 317, 83 (2008).

[30] S.K. Tripathy, et al.: Astrophys Space Sci. 315, 105 (2008).

[31] V.U.M. Rao, D.C. Papa Rao: Astrophys Space Sci 357, 77 (2015).

[32] G.K. Goswami, et al.: Astrophys Space Sci 361, 47 (2016).

[33] P.K. Sahoo, et al.: Eur. Phys. J. Plus 131, 333 (2016).

[34] C.W. Misner: Nature 214, 40 (1967).

[35] C.W. Misner: Astrophys. J. 151, 431 (1968).

[36] J.D. Barrow: Phys. Lett. B 180, 335 (1986).

[37] T. Padmanabhan, S.M. Chitre: Phys. Lett. A 120, 433 (1987).

[38] D. Pavon, J. Bafluy, D. Jou: Class. Quantum gravity, 8, 347 (1991).

[39] J.A.S. Lima, A.S.M. Germano, L.R.W. Abrama: Phys. Rev. D 53, 4287 (1993).

[40] R. Martens: Class. Quantum gravity 12, 1455 (1995).

[41] R. Bali, A. Pradhan: Chin. Phys. Lett. 24, 585 (2007).

[42] S.K. Tripathy, et al.: Astrophys. Space Sci. 321, 247 (2009).

[43] G.P. Singh, A.Y. Kale: Astrophys. Space Sci. 331, 207 (2011).

[44] V.U.M. Rao, K.V.S. Sireesha: Int. J. Theor. Phys. 51, 3013 (2012).

[45] Shri Ram, M.K. Singh, M.K. Verma: Journal of Modern Physics 3, 9 (2012).

[46] V.U.M. Rao, et al.: Prespacetime J. 6, 531 (2015).

[47] V.U.M. Rao, et al.: The African Review of Physics 10, 0060 (2015). 
[48] K.S. Adhav: Int J Theor Phys 48, 700 (2009).

[49] V.U.M. Rao, et al.: Astrophys Space Sci 335, 635 (2011).

[50] T.V. Sagar, et al.: Astrophys Space Sci. 349, 467 (2014).

[51] M.S. Berman: Nuovo Cimento B 74, 182 (1983).

[52] C.B. Collins, et al.: Gen. Relativ. Gravit. 12, 805 (1980).

[53] T. Chiba, et al.: Phys. Rev. D 75, 124014 (2007).

[54] V.B. Johri, R. Sudharsan: Aust. J. Phys., 42, 215 (1989).

[55] K. Uddin, et al.: Class. Quantum Grav. 24, 3951 (2007).

[56] G.L. Murphy: Phys. Rev. D 8, 4231 (1973). 